\title{
THE RECKONING OF CERTAIN QUARTIC AND OCTIC GAUSS SUMS
}

\author{
by BRUCE C. BERNDT and S. CHOWLA
}

(Received 2 February, 1976)

In this brief note, we evaluate certain quartic and octic Gauss sums with the use of theorems on fourth and eighth power difference sets. We recall that a subset $H$ of a finite (additive) abelian group $G$ is said to be a difference set of $G[5$, p. 64] if for some fixed natural number $\lambda$, every nonzero element of $G$ can be written as a difference of two elements of $H$ in exactly $\lambda$ ways.

Throughout the paper, $p$ designates an odd prime. An evaluation of general quartic Gauss sums for $p \equiv 1(\bmod 4)$ can be found in Hasse's book [3, pp. 490-493]. R. J. Evans and the first named author [1] have explicitly evaluated general octic Gauss sums for $p \equiv 1(\bmod 8)$. Unfortunately, only a special class of Gauss sums can be evaluated by the method of this note, but we feel that the method's brevity and simplicity are worth noting.

For $m \in\{4,8\}$ and $p \equiv 1(\bmod m)$, let

$$
S_{m}(p)=\sum_{r} e^{2 \pi i r / p},
$$

where the sum is over the $(p-1) / m$ distinct $m$ th power residues $(\bmod p)$.

THEOREM 1. Assume that $p=4 b^{2}+1$, where $b$ is odd. Then

$$
S_{4}(p)=\frac{\sqrt{p}-1}{4} \pm \frac{i}{2} \sqrt{\frac{p+\sqrt{p}}{2}} .
$$

Proof. For the primes under consideration, the latter named author [2], [5, p. 92] has shown that the $(p-1) / 4$ quartic residues modulo $p$ form a difference set. Hence,

$$
S_{4}(p) \overline{S_{4}(p)}=\sum_{r} e^{2 \pi i r / p} \sum_{r} e^{-2 \pi i r / p}=\frac{p-1}{4}+\frac{p-5}{16} \sum_{n=1}^{p-1} e^{2 \pi i n / p}=\frac{3 p+1}{16} .
$$

For the number of terms in the product at the far left side of $(1)$ is $\{(p-1) / 4\}^{2}$, and so, since the $(p-1) / 4$ quartic residues $(\bmod p)$ form a difference set, a simple calculation shows that each integer $n, 1 \leqq n \leqq p-1$, must occur precisely $(p-5) / 16$ times. Since $p \equiv 5(\bmod 8),-1$ is a quadratic residue but a quartic nonresidue modulo $p$. Thus, if $r_{1}, \ldots, r_{k}, k=(p-1) / 4$, denote a complete set of quartic residues modulo $p$, then $\pm r_{1}, \ldots, \pm r_{k}$ represent the quadratic residues modulo $p$. Thus, letting $r$ run through the distinct quadratic residues modulo $p$, we have

$$
S_{4}(p)+\overline{S_{4}(p)}=\sum_{r} e^{2 \pi i r / p}=\frac{1}{2}(\sqrt{p}-1)
$$

where we have used the well-known evaluation of quadratic Gauss sums [3, p. 115]. Solving (1) and (2) simultaneously for $S_{4}(p)$, we obtain the desired result.

Glasgow Math. J. 18 (1977) 153-155. 
THEOREM 2. Assume that $p=4 b^{2}+9$, where $b$ is odd. Then

$$
S_{4}(p)=\frac{\sqrt{p}-1}{4} \pm \frac{i}{2} \sqrt{\frac{p-3 \sqrt{p}}{2}}
$$

Proof. By a theorem of E. Lehmer [4], [5, p. 92], for the primes under consideration, the $(p-1) / 4$ quartic residues and zero form a difference set. Hence,

$$
\left.\left(S_{4}(p)+1\right) \widehat{\left(S_{4}(p)\right.}+1\right)=\frac{p+3}{4}+\frac{p+3}{16} \sum_{n=1}^{p-1} e^{2 \pi i n / p}=\frac{3 p+9}{16} .
$$

By the same argument as in the previous proof, (2) again holds. Solving (2) and (3) simultaneously for $S_{4}(p)$, we complete the proof of the theorem. Then

THEOREM 3. Assume that $p=8 b^{2}+1$, where $b$ is odd, and that $p=64 c^{2}+9$, where $c$ is odd.

$$
S_{8}(p)=\frac{1}{4}\left\{\frac{\sqrt{p}-1}{2}+\varepsilon \sqrt{\frac{p+3 \sqrt{p}}{2}} \pm \sqrt{(\sqrt{p}-1)\left\{\varepsilon \sqrt{\frac{p+3 \sqrt{p}}{2}}-\sqrt{p}\right\}}\right\},
$$

where $\varepsilon= \pm 1$.

Proof. By a theorem of E. Lehmer [4], for the primes considered here, the $(p-1) / 8$ octic residues modulo $p$ form a difference set. Hence,

$$
S_{8}(p) \overline{S_{8}(p)}=\frac{p-1}{8}+\frac{p-9}{64} \sum_{n=1}^{p-1} e^{2 \pi i n / p}=\frac{7 p+1}{64} .
$$

Since $p \equiv 9(\bmod 16),-1$ is a quartic residue but an octic nonresidue modulo $p$. Hence, arguing as in the proof of Theorem 1 , we find that

$$
S_{8}(p)+\overline{S_{8}(p)}=S_{4}(p)
$$

However by [3, pp. 490-493], [1],

$$
S_{4}(p)=\frac{\sqrt{p}-1}{4}+\varepsilon \frac{1}{2} \sqrt{\frac{p+3 \sqrt{p}}{2}},
$$

where $\varepsilon= \pm 1$. Substituting (6) into (5) and then solving (4) and (5) simultaneously, we reach the desired result.

E. Lehmer [4] has also determined necessary and sufficient conditions for zero and the eighth powers modulo $p$ to form a difference set. By using the ideas in the proofs of Theorems 2 and 3, we may also evaluate octic Gauss sums for these primes.

In [1], the opposite tack is taken from that presented here; the theory of Gauss sums is used to prove theorems on difference sets. 


\section{REFERENCES}

1. Bruce C. Berndt and Ronald J. Evans, manuscript in preparation.

2. S. Chowla, A property of biquadratic residues, Proc. Nat. Acad. Sci. India, Sect. A 14 (1944), 45-46.

3. Helmut Hasse, Vorlesungen über Zahlentheorie (Springer-Verlag, 1964).

4. E. Lehmer, On residue difference sets, Canad. J. Math. 5 (1953), 425-432.

5. Henry B. Mann, Addition theorems (Wiley, 1965).

UNIVERSITY OF ILLINOIS

URBANA

ILLINOIS 61801

U.S.A.
INSTITUTE FOR ADVANCED Study PRINCETON

NEW JERSEY 08540

U.S.A. 